\title{
ANALYSIS OF A SHORT PERIODIC PULSATOR: SX PHOENICIS STAR XX CYG
}

\author{
Mohamed Abdel-Sabour ${ }^{1}$, Ahmed Shokry ${ }^{1}$, and Ahmed Ibrahim ${ }^{2,3}$ \\ Received May 5 2020; accepted August 72020
}

\begin{abstract}
Photometric observations were made of the SX Phoenicis star XX Cyg between September and October 2019, using the 1.88-m Kottamia reflector telescope in Egypt. We used 340 CCD observations with blue-visible-red (BVR) filters to derive light curves. In addition, we obtained 9540 visual magnitudes for XX Cyg from the literature to prepare an observed-minus-calculated (O-C) diagram. 85 new times of maximum for XX Cyg are presented. We did not detect a bump in the descending portion of the light curve of maximum light for XX Cyg. However, we did detect a secular bump in the phased light curves, which changes with phase in some SuperWASP observations. We found the change in period of XX Cyg to be $\mathrm{d} P / \mathrm{d} t=15.5 \times 10^{-5} \mathrm{~s} / \mathrm{yr}$, with its amplitude decreasing at a rate of $0.7 \mathrm{mmag} /$ year. Stellar parameters of XX Cyg and its position in the instability strip of the Hertzsprung Russell stellar evolution diagram are presented.
\end{abstract}

\section{RESUMEN}

Se realizaron observaciones fotométricas de la estrella tipo SX Phoenicis XX Cyg entre septiembre y octubre de 2019 con el telescopio de 1.88 m Kottamia, en Egipto. Mediante 340 observaciones con CCD en los filtros BVR derivamos curvas de luz. También obtuvimos 9540 magnitudes visuales para XX Cyg de la literatura, para preparar un diagrama O-C. Presentamos 85 nuevos tiempos del máximo para XX Cyg. No detectamos una protuberancia en la parte descendiente del máximo de la curva de luz de XX Cyg. Sin embargo, encontramos una protuberancia secular en las curvas de luz en fase, la cual cambia con la fase en algunas observaciones del SuperWASP. Encontramos que el cambio del período de XX Cyg es $\mathrm{d} P / \mathrm{d} t=15.5 \times 10^{-5} \mathrm{~s} / \mathrm{yr}$, y que la amplitud decrece a una tasa de $0.7 \mathrm{mmag} /$ year. Presentamos los parámetros estelares y el estado evolutivo de XX Cyg, que se encuentra en la franja de inestabilidad del diagrama de Hertzsprung Russell.

Key Words: stars: evolution — stars: individual: XX Cyg — stars: variables: general

\section{INTRODUCTION}

The evolution of stars with 0.9 to $120 M_{\odot}$ is well described theoretically by Schaller et al. (1992). After leaving the main sequence, they evolve to the right in the Hertzsprung-Russell (H-R) diagram. Then, they pass through the instability strip and evolve toward cooler temperatures. The rate of period change for pulsating variable stars is of great value for establish-

\footnotetext{
${ }^{1}$ National Research Institute of Astronomy and Geophysics (NRIAG), Cairo, Egypt.

${ }^{2}$ Dept. of Astronomy and Meteorology, Faculty of Science, Al-Azhar University, Nasr City, Cairo, Egypt.

${ }^{3}$ King Saud Univ, Dept. Phys \& Astron, Coll. Sci, Saudi Arabia.
}

ing both their evolutionary status and their position in the instability strip, as well as for testing stellar evolutionary models (Turner et al. 1998).

SX Phoenicis variables are analogues of Delta Scuti stars and are located below the classical Cepheid variable stars in the instability strip, closer to the main sequence. In general, SX Phoenicis variables have periods ranging between 30 minutes and six hours. SX Phe stars are interesting because they often pulsate in so-called non-radial modes, they have a lower luminosity and are more metal poor than Delta Scuti stars (Frolov,1974). 


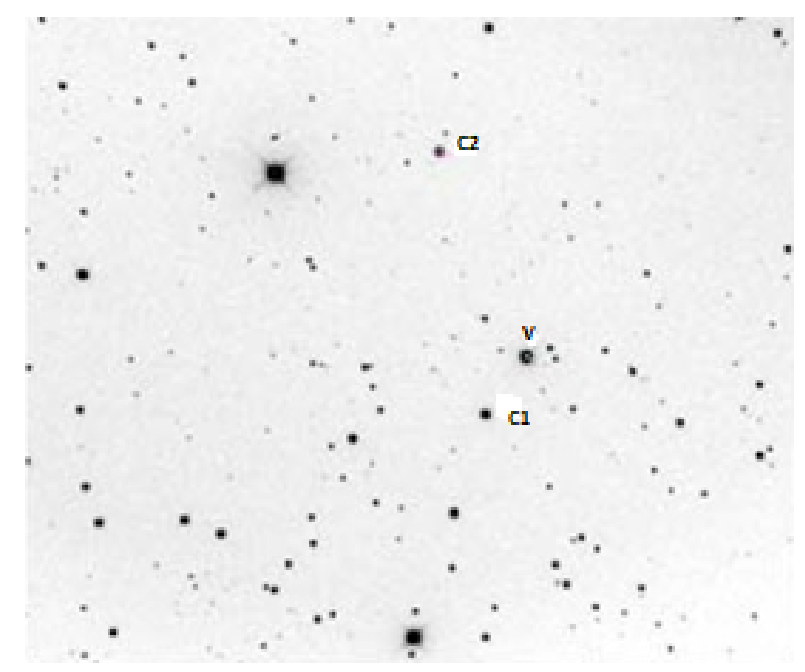

Fig. 1. XX Cyg variable star $(V)$ with comparison star (C1) and check star (C2), as seen from the CCD camera at $\mathrm{KAO}$.

XX Cyg is a Population-II variable star, which has a fundamental radial mode, excited with a high velocity $(V)=-108 \mathrm{~km} / \mathrm{s}$; Joner 1982); see Figure 1. It has a visual magnitude of $V=11.7 \mathrm{mag}$ with an amplitude in this band of $\Delta V=0.80 \mathrm{mag}$ (Kiss and Derakas 2000) and a radial-velocity amplitude of $37 \mathrm{~km} / \mathrm{s}$ (Joner 1982). Its classification changed from a dwarf Cepheid (Joner 1982, McNamara and Feltz 1980) to the more modern classification as a Population-II SX Phoenicis-type variable star. Our study examines the amplitude stability of XX Cyg and investigates the dependence of the pulsation amplitude on its location within the instability strip.

\section{OBSERVATIONS AND DATA REDUCTION}

\subsection{Observations}

To investigate the variability of our target star (XX Cyg), photometric observations from the archives of the All-Sky Automated Survey for Supernovae (ASAS-SN), Wide Angle Search for Planets (SuperWASP), and American Association of Variable Star Observers (AAVSO) provided new maximum-light times for our target. The data, span a time interval of over 110 years, from the early twentieth century to the present, as shown in the references. We acquired four nights' observations on September 24/25 and October 8/9, 9/10, and 20/21, 2019, as displayed in Figure 2. The observations were obtained through standard Johnson blue-visible-red $(B V R)$ filters; and the exposure times ranged from 20 to 60 seconds, depending on the atmospheric conditions and background

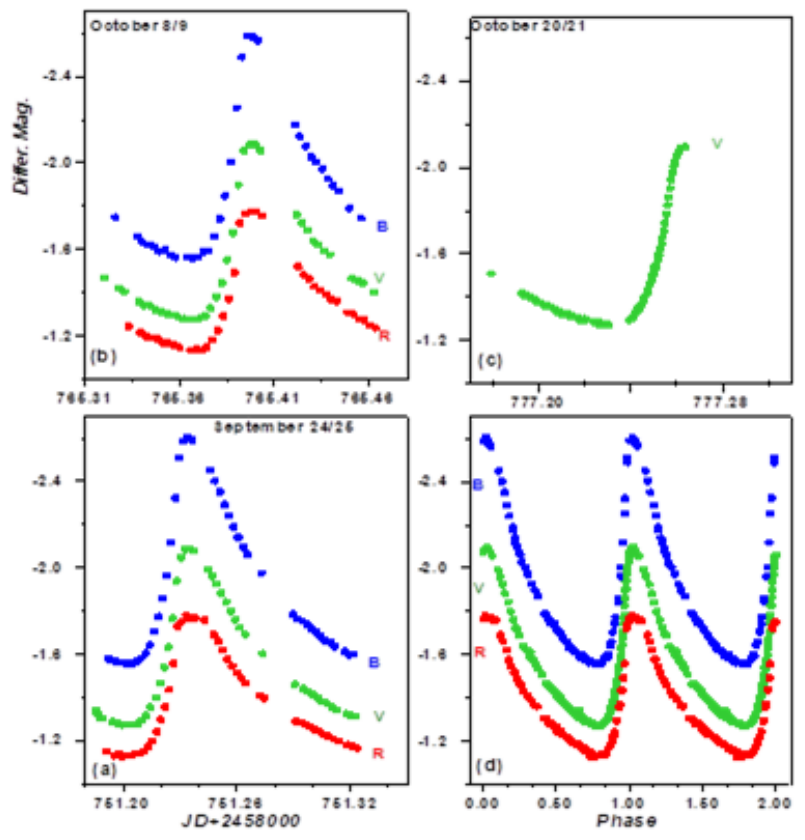

Fig. 2. Differential instrumental magnitude in $B V R$ colors $(\Delta B, \Delta V$, and $\Delta R)(\mathrm{a}, \mathrm{b}, \mathrm{c})$ and the phases in three colors (d) of XX Cyg. The color figure can be viewed online.

contribution. All observations from the Kottamia Observatory (KAO) were made using a 2048x2048pixel EEV CCD 42-40 camera, cooled by liquid nitrogen and attached to the Newtonian focus of the 1.88-m Kottamia reflector telescope in Egypt. The Kottamia Observatory is located at a latitude of $29^{\circ} 56^{\prime} 02.43^{\prime \prime} \mathrm{N}$, a longitude of $31^{\circ} 49^{\prime} 40.1^{\prime \prime} \mathrm{E}$, and a height of $467 \mathrm{~m}$. Figure 1 shows one of the $V$-band images of the variable XX Cyg, along with comparison and check stars. The comparison star has coordinates $\alpha_{2000}=20^{\mathrm{h}} 03^{\mathrm{m}} 18^{\mathrm{s}}$ and $\delta_{2000}=+58^{\circ} 55.6^{\prime} 6.9^{\prime \prime}$. All times were corrected to Heliocentric Julian Date (HJD). Standard data reduction processes (i.e., dark and bias removal, flatfield correction) and aperture photometry have been applied to the present observations, using the free data reduction software (MuniWin) from the website (http://c-munipack.sourceforge.net/). We obtained differential instrumental magnitudes for each filter and constructed the present light curves of the system XX Cyg.

\subsection{Methods of Reduction}

We used two methods to determine the period and amplitude variations. The first one originated with Hertzsprung (Hertzsprung,1928) and the second one is the Fourier decomposition method. Modern usage 


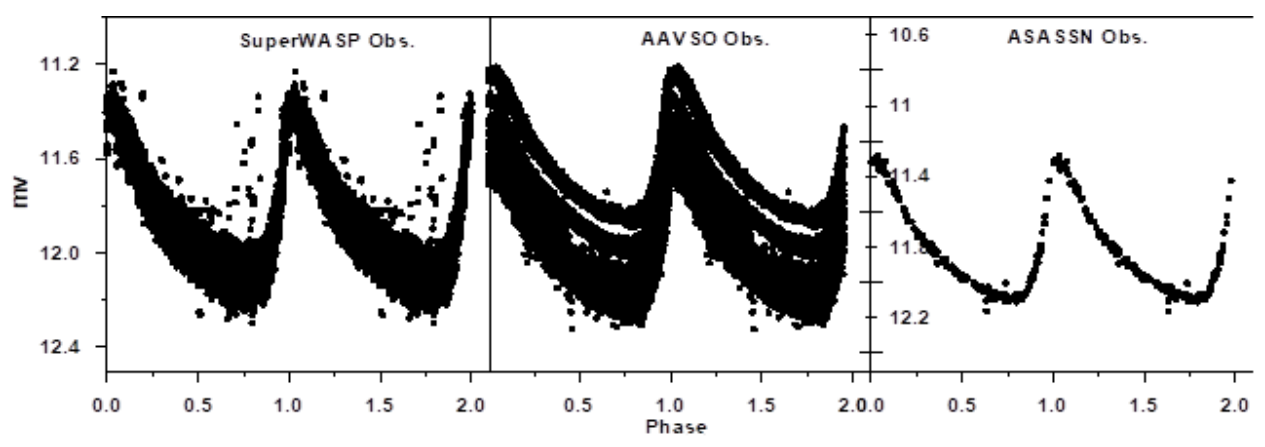

Fig. 3. Phase observations from the ASAS, AAVSO, and SuperWASP archives.

of the first method has been described by Berdnikov (1992), Turner and Berdnikov (2001), and Turner (2003). The observed-minus-calculated (O-C) technique is used to study changes in the periods of Cepheids with high accuracy. The phased data were used to construct seasonal light curves, which were matched to a standard light curve to detect phase shifts, indicating a period change. An alternative procedure used by Abdel-Sabour et al. (2015) relies on a set of high-quality light curves as a standard, to which independent data sets are matched in magnitude and phase space using least-squares techniques. In the second method, we use Fourier decomposition (FD) as a tool for variable-star diagnostics. As the light curves of pulsating variable stars are periodic, they can be written as shown in the following equations;

$$
f(x)=A_{0}+\sum_{n=i}^{N} A_{n} \cos \left(i \omega\left(t_{0}-t_{i}\right)+\varphi_{i}\right) .
$$

Or

$$
\begin{aligned}
f(x)= & A_{0}+\sum_{n=i}^{N} A_{n} \cos \left(i \omega\left(t_{0}-t_{i}\right)\right) \\
& +\sum_{n=i}^{N} B_{n} \sin \left(i \omega\left(t_{0}-t_{i}\right)\right),
\end{aligned}
$$

where $A_{i}=\sqrt{(}\left(a^{2}+b^{2}\right), \tan \Phi_{i}=-b_{i} / a_{i}$. Because the period is known from the respective databases, the observation time can be folded into the phase $\left(\varphi_{i}\right)$ (Ngeow et al. 2003).

The relative Fourier parameters are $\varphi_{i 1}=\varphi_{i}-i \varphi_{1}$ and $R_{i 1}=A_{i} / A_{1}$, where $\mathrm{i}>0$. We used the above equations to calculate the Fourier parameters from 85 light curves of XX Cyg. The combination of coefficients, $R_{i 1}$ and $\varphi_{i 1}$, where $\mathrm{i}=2,3,4 \ldots$, can be used to describe the progression of the light-curve shape, as seen in Figure 5 for the visual amplitude $A_{v}$. We can also use these coefficients for variable-star classification (Deb and Singh 2009).

\section{RESULTS AND DISCUSSION}

\subsection{O-C Light Curve and Period Change of $X X$ Cyg}

Comparing the observed time with the calculated time of maximum brightness (O-C method) is the classical method for studying period changes in variable stars, because it is sensitive to the accumulated effects of the period changes. We used all of the data published in the literature to fill the gaps in the O$\mathrm{C}$ diagram. Light curves with a large scatter in the observations were removed from our analysis. i.e. If the scatter in the phased light curve was about the same as the scatter in the raw data (usually about $0.2 \mathrm{mag}$. or less) we used it in the O-C diagram. All of the maximum-light times used to study the period change of XX Cyg are presented in Figure 4. The computed time of maximum was measured using the linear ephemeris of Szeidl and Mahdy (1981). The time of maximum brightness $\left(H J D_{M a x}\right)$ can be modeled as a function of epoch $(E)$ using the following quadratic equation:

$$
H I D_{\max }=M_{0}+P E+Q E^{2} .
$$

Here, $M_{0}$ is a new epoch, $P$ is the new period, and $Q$ is used to measure the period change $(d P / d t)$ in seconds per year given by; $d P / d t=(2 Q / P)(32,557,600) \mathrm{s} / \mathrm{yr}$. The evolution of the period over time was investigated by means of the classic O-C method and Fourier transforms, as mentioned in the last section. The O-C behavior of XX Cyg was observed from November 1905 to October 2019. A fit to the O-C diagram was made using a quadratic polynomial, which corresponds to a constantly changing period. After analysis, we found the following ephemeris:

$H J D_{\max }=2430671.103-0.1349 E-2.82 \times 10^{-13} E^{2}$. 

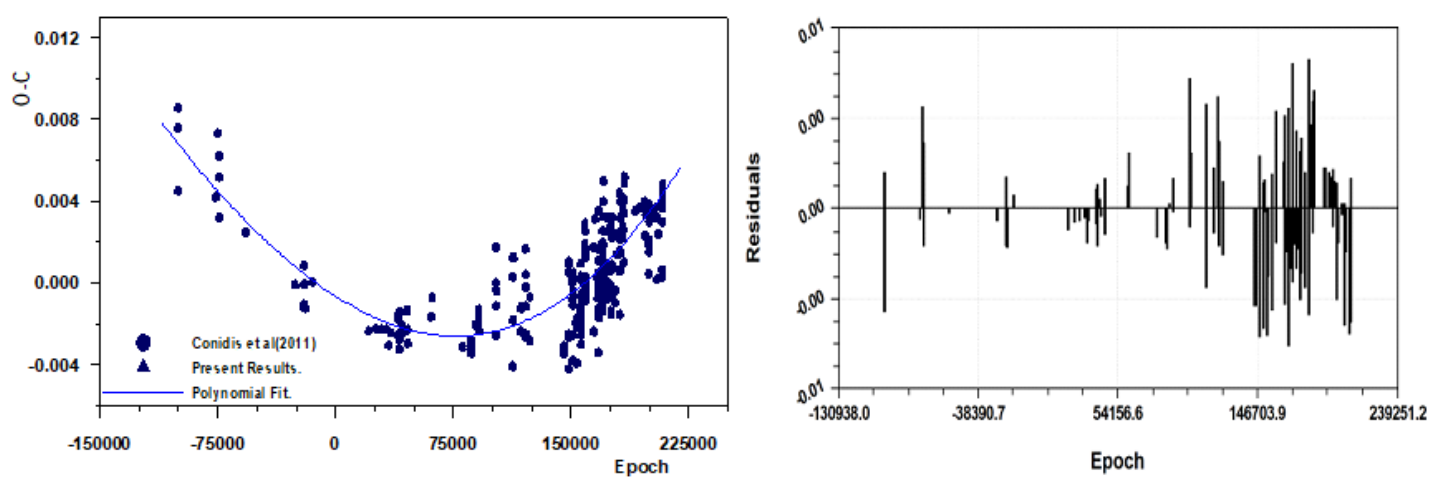

Fig. 4. O-C diagram for XX Cyg and the best fitted parabola, plotted as a function of the observed maximum-light epoch (left panel) and the O-C residuals (right). The color figure can be viewed online.

The parabolic trend in the $\mathrm{O}-\mathrm{C}$ data reflects a regular period decrease or increase. From this analysis we find that the period is increasing at a rate of $d P / d t=(15.50 \pm 1.10) \times 10^{-5} \mathrm{~s} / \mathrm{yr}$ or $([1 / P] d P / d t=$ $\left.1.33 \times 10^{-8} \mathrm{yr}^{-1}\right)$. Our rate of period change agrees with an earlier estimate by Conidis et al. (2011) $\left(1.337 \times 10^{-8} \mathrm{yr}^{-1}\right.$.) but differs slightly from Blake, et al. $(2003)\left(1.13 \times 10^{-8} \mathrm{yr}^{-1}\right)$ because we used extra data at recent epochs, including our KAO observations.

\subsection{Amplitude Variation in XX Cyg}

Variations in the amplitude, period, and shape of the light curves are known as the Blazhko Effect. This phenomenon can be seen easily in RR Lyrae variables. A frequency analysis of XX Cyg was performed with a package of computer programs, employing single-frequency (Fourier) and multiple-frequency least-squares techniques (PERIOD04, Breger 1990). The technique fits a number of simultaneous sinusoidal variations in the magnitude domain and does not rely on prewhitening. We analyzed the stellar light curves using the PERIOD04 program and FORTRAN programs to evaluate the Fourier parameters for all available observations. The amplitude behavior in the visible band is presented in Figure 5. Linearly fitting the variations resulted in the following equation: $A_{V}=0.1165^{m}-\left(7.544^{m} \times 10^{-7}\right)$ Epoch, which indicated a decreasing rate of $0.7 \mathrm{mmag} / \mathrm{yr}$. If we use only our recent observations $(\mathrm{E}>50000)$ we find approximately the same results, $0.56 \mathrm{mmag} / \mathrm{yr}$, as presented in Figure 5. The results for the Fourier amplitudes agree with those derived using wavelet analysis by Zhou et al. (2002). These results indicate that there is a relation between pulsation amplitude and rate of period change.

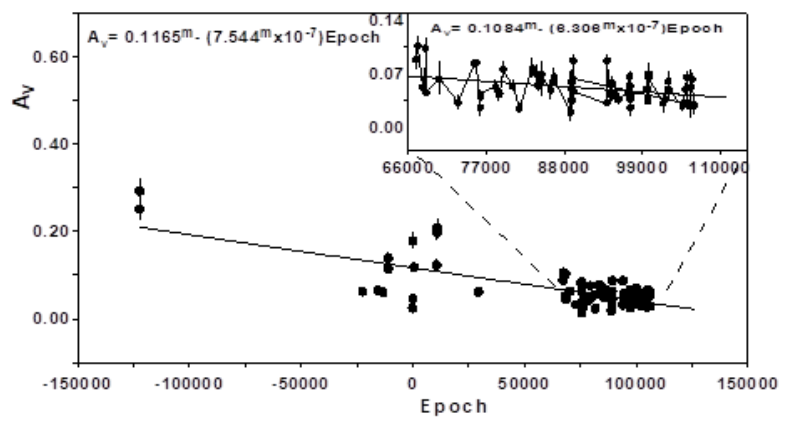

Fig. 5. Time evolution for the AV amplitude of the fundamental frequency of XX Cyg, determined by Fourier analysis. The trend of the variations is shown by a linearfitted line. The inset shows a fit to only epochs $\mathrm{E}>50,000$ and shows approximately a similar decreasing trend.

\subsection{Ambiguity in the Light Curves}

When we tried to test the amplitude stability of our system we noticed a damping in the light curves in some observations, as presented partially in Figure 6 , from phase 0.1 to 0.8 . These observations are from the SuperWASP Camera\#145 on nights 2454613.59, $2454629.565,2454631.54,2454632.54,2454659.46$, and 2454660.46 . These may be the same light curve bumps described by Blake et al. (2003) and Sadun and Ressler (1986). However, we did not detect the bump in our BVR observations. Also, we failed to observe similar bumps in the other automated surveys (Northern Sky Variability Survey (NSVS), Hipparcos Catalogue (HIP), Kamogata/Kiso/Kyoto Wide-field Survey (KWS), and ASAS SN). The SuperWASP bump detections are significant because the bump amplitudes are more than three times larger than the photometric errors of the dataset. Also, we noticed the positions and effects of the bumps changes with phases. i.e. a smaller bump 

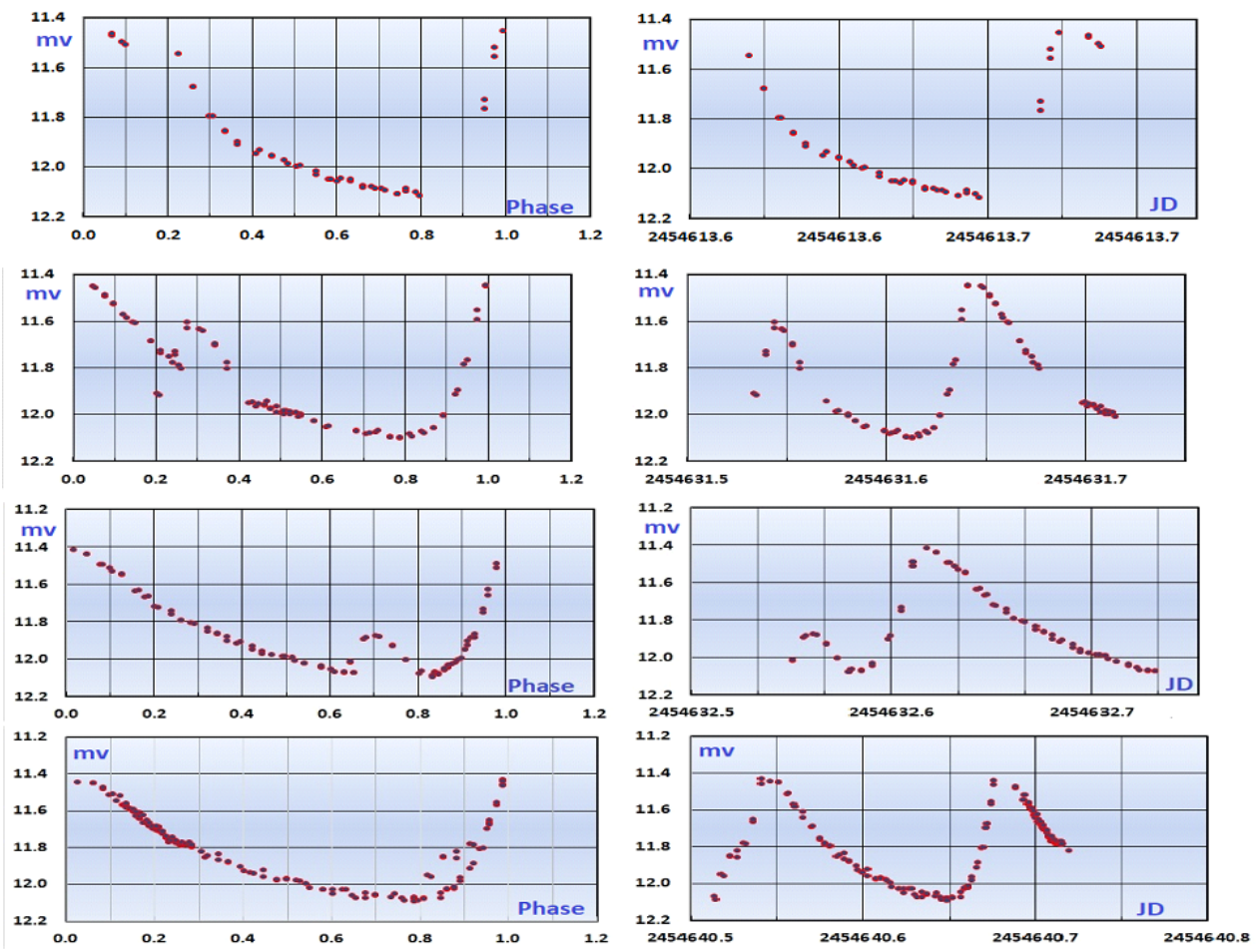

Fig. 6. Six nights: 2454613.59, 2454631.54, 2454632.54, and 2454640.46 Light curves and phases from SuperWASP observations with Camera\#145. The color figure can be viewed online.

with a dip of amplitude approximately 0.1 magnitude, which peaks at a phase of 0.15 and a larger bump occurring with ascending phases towards the brightness minimum (as seen at one phase in Figure 6). This damping in the light curve or bump in phase may be a result of magnetic activity or shock waves (Gillet, D. 1988, Balona et al. 2015).

\section{ABSOLUTE PARAMETERS OF SYSTEM XX CYG}

Spectroscopic observations are an important source for estimating physical parameters. At present, no spectroscopic observations are available for XX Cyg except for the radial velocities reported by Joner (1982), which yield a mean $R V$ of $-108 \mathrm{~km} / \mathrm{s}$. So, the absolute parameters cannot be determined directly. Therefore, we used simple approximation formulae (Harmanec, 1988), which relate the effective temperature to other basic physical parameters (mass, radius, luminosity, and bolometric magnitude).

\subsection{Physical Parameters and Evolutionary State of $X X$ Cyg}

If the effective temperature $\left(T_{\text {eff }}\right)$ and bolometric magnitude $\left(M_{b o l}\right)$ are known, as well as the period and pulsation mode, the pulsation mass $\left(M_{\text {puls }}\right)$ follows directly from the fundamental equation of stellar pulsation, $\left.Q=\sqrt{(} M / R^{2}\right)$, where $P$ is the period in days, $M$ and $R$ are the mass and radius in solar units, respectively, and $Q$ is the pulsation constant. The physical parameters, absolute magnitude $\left(M_{V}\right)$, relative radius $\left(R / R_{\odot}\right)$, relative luminosity $\left(L / L_{\odot}\right)$, surface gravity $(g)$, and pulsation constant $(Q)$, were calculated using standard stellar-structure equations (Gray 2005 equation 3, Reed Cameron 1998, Collier Cameron et al. 2011, and Breger 1990) assuming the effective temperature of XX Cyg $\left(T_{\text {eff }}\right.$ $=7530 \mathrm{~K})$ from Zhou et al. (2002), $\log g_{\odot}=4.44$, and $\mathrm{Mbol}_{\odot}=4.74$. To see the location of XX Cyg in the Hertzsprung-Russell diagram, we calculated the parameters using the equations of Reed (1998), Torres (2010), and Flower (1996). The results are presented in Table 1.

These results are in good agreement with a thesis by Bowman (2016) (see his Figure 4.12 for lowfrequency pulsations, $\left.\nu \leq 25 d^{-1}\right)$. The evolu- 


\section{TABLE 1}

PHYSICAL PARAMETERS: MASS, LUMINOSITY, RADIUS, BOLOMETRIC MAGNITUDE, STELLAR GRAVITY, AND AGE OF XX CYG

\begin{tabular}{ccccccc}
\hline Ref. & $M / M_{\odot}$ & $L / L_{\odot}$ & $R / R_{\odot}$ & $M_{\text {bol }}$ & $\log g$ & Age $(\mathrm{yr}) \times 10^{9}$ \\
\hline Our results & 1.955 & 1.038 & 1.944 & 1.688 & 4.086 & 0.949 \\
& $(0.164)$ & $(0.026)$ & $(0.343)$ & $(0.164)$ & $(0.079)$ & $(0.064)$ \\
Zhou (2002) & $1.0(0.5)$ & & 2.400 & 1.700 & 3.660 & 1.00 \\
Yang et al. (2012) & $1.7(0.1)$ & & & 1.700 & & $0.90(0.30)$ \\
\hline
\end{tabular}
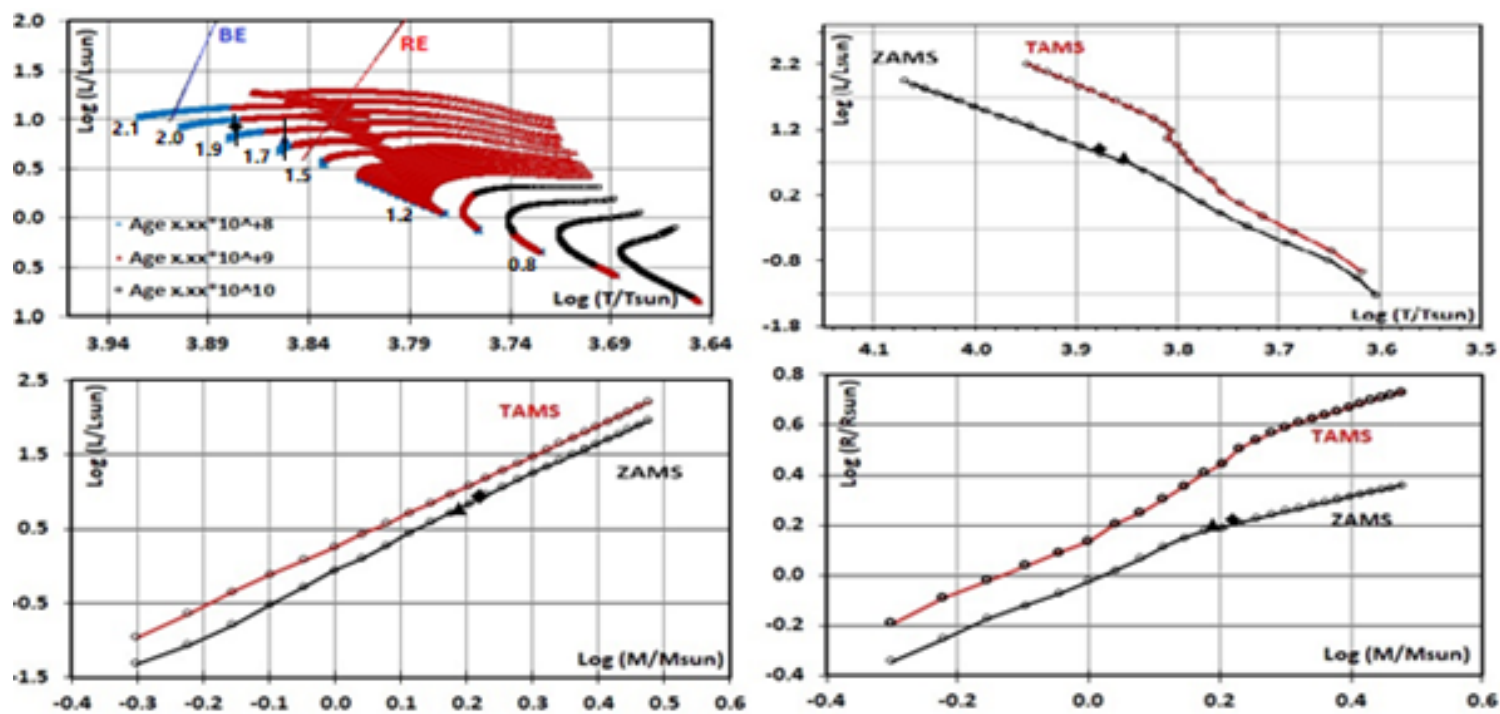

Fig. 7. Position of XX Cyg (square) and the Cepheid variable GSC 02444-00241 (triangle) in temperature-luminosity (HR diagram), mass-luminosity and mass-radius diagrams. The top left plot shows theoretical mass tracks from Mowlavi et al. (2012) for $\mathrm{Z}=0.014$ over a range of ages (in Gyr). The blue (BE) and red (RE) edges of the instability strip are marked. The color figure can be viewed online.

tionary tracks for the non-rotating model, as computed by Mowlavi et al. (2012), for both zeroage main-sequence stars (ZAMS) and terminal-age main-sequence stars (TAMS) with a metallicity of $\mathrm{Z}=0.014$ (solar metallicity), are presented in Figure 7. The absolute physical parameters of XX Cyg, are plotted as mass-luminosity $(M-L)$, mass-radius $(M-R)$, and H-R diagrams at Figure 7 . The parameters are in good agreement with those expected for SX Phe variables in the $\log M-\log L$ plane. XX Cyg is close to, or on, the ZAMS in all the diagrams of Figure 7 and lies halfway between the blue edge (BE) and red edge (RE) of the instability strip, as shown in the $L-T$ relation (H-R diagram). To estimate the age of the system, we plotted theoretically calculated models (Mowlavi et al. 2012) for temperature versus luminosity ( $T-L)$ (Figure 7). From this diagram, and from McNamara and Feltz (1980), the age of XX
Cyg should be approximately $(0.949 \pm 0.064) \times 10^{9}$ years, It is close to the age predicted by Zhou (2002) and Yang et al. (2012), which is $(0.9 \pm 0.3) \times 10^{9}$ years as shown in Table 1. The location of XX Cyg in the H-R diagram is shown in Figure 7. These parameters would explain the position of the star at or near the main sequence (Rodriguez and Breger 2001).

\section{CONCLUSION}

The following conclusions were drawn:

1. Photometric observations from the archives of the ASAS-SN, SuperWASP and AAVSO provided 85 new times of maximum-light for XX Cyg. 
2. From the O-C result (Figure 4), we estimated the period change rate for XX Cyg; the result was $(\mathrm{d} P / \mathrm{d} t)=(15.248 \pm 1.092) \times 10^{-5} \mathrm{~s} / \mathrm{yr}$ and/or $(1 / P) \mathrm{d} P / \mathrm{d} t=1.33 \times 10^{-8} \mathrm{yr}^{-1}$.

3. We also found that the amplitude was decreasing over time.

4. The amplitude behavior in the visible band is decreasing at a rate of 0.7 mmag per year. This variation of amplitude is not at all understood and is one of the open questions about SX Phe stars.

5. As noticed from the period change and amplitude variations, there seems to be a correlation between the two quantities: the amplitude decreases with increasing period.

6. The existence of a bump in the light curve is still uncertain. However, a bump in the amplitude was observed in an automated SuperWASP survey, as noted in the text. Maybe there are large magnetically active regions on the surface, or shock waves from the stellar interior, which are responsible for the bump.

7. Multi-wavelength and radial-velocity observations of XX Cyg are required to determine the reason for this bump.

8. We calculated the physical parameters for $\mathrm{XX}$ Cyg, and the results were found as $L / L_{\odot}=$ 1.038(0.026), $M / M_{\odot}=1.955(0.164), R / R_{\odot}=$ 1.944(0.343), $\log g=4.086$; in addition to an age $(\tau)$ equal to $0.949(0.064) \times 10^{9}$ year.

The authors would like to extend their sincere appreciation to the Deanship of Scientific Research (DSR) and College of Science Research Center at King Saud University, Saudi Arabia for their financial support. Also, the authors are thankful to the Egyptian Science and Technology Development Fund (STDF) for funding the Kottamia Faint Imaging Spectro-Polarimeter (KFISP), STDF 2531 and the Kottamia Center of Scientific Excellence for Astronomy and Space Science (KCScE), STDF 5217, as well as the support of the KAO team and the funding from NRIAG, Egypt. Also, we would like to thank Dr. A. Essam and Dr. M. Nouh for enlightening discussions.

\section{REFERENCES}

Abdel-Sabour, M., Ibrahim, A., Essam, A., \& Elkhateeb, M. M. 2015, RoAj, 25, 157

Balona, L. A., Broomhall, A.-M., \& Kosovichev, A., et al. 2015, MNRAS, 450, 956

Berdnikov, L. N. 1992, A\&AT, 2, 43

Blake, R. M., Delaney, P., Khosravani, H., Tome, J., \& Lightman, M. 2003, PASP, 115, 212

Bowman, D. M. 2016, Amplitude Modulation and Energy Conservation of Pulsation Nodes in Delta Scuti Stars, PhD, Thesis, University of Central Lancashire, UK, 267B

Breger, M. 1990, ASPC, 11, 263

Collier, C. A., Wilson. D. M., West, R. G., Hebb, L. et al. 2007, MNRAS, 380, 1230

Deb, S. \& Singh, H. P. 2009, A\&A, 507, 1729

Eddington, A. S. and Plakidis, S. 1929, MNRAS, 90, 65

Flower, P. J. 1996, ApJ, 469, 355

Frolo, M. S. 1974, Sci. Inform. Astron. Council USSR Acad. Sci., 33, 29

García, J. 2012, JAAVSO, 40, 272

Gillet, D. \& Crowe, R. A. 1988, A\&A, 199, 242

Gray, D. F. 2005, The Observation and Analysis of Stellar Photospheres, (Cambridge, UK: CUP)

Harmanec, P. 1988, AICz, 39, 329

Hertzsprung, E. 1928, BAN, 4, 178

Joner, M. D. 1982, PASP, 94, 289

Kiss, L. L. \& Derekas, A. 2000, IBVS, 4950, 1

McNamara, D. H. \& Feltz, K. A. Jr. 1980, PASP, 92, 195

Mowlavi, N., Eggenberger, P., Meynet, G., et al. 2012, A\&A, 541, 41

Ngeow, C. C., Kanbur, S. M., Nikolaev, S., Tanvir, N. R., \& Hendry, M. A. 2003, ApJ, 586, 959

Reed, B. C. 1998, JRASC, 92, 36

Rodríguez, E. \& Breger, M. 2001, A\&A, 366, 178

Sadun, A. C. \& Ressler, M. 1986, PASP, 98, 504

Schaller, G., Schaerer, D., Meynet, G., \& Maeder, A. 1992, A\&AS, 96, 269

Szeidl, B. \& Mahdy, H. A. 1981, MiUng, 75, 1

Torres, G. 2010, AJ, 140, 1158

Turner, D. G. \& Berdnikov, L. N. 2001, OAP, 14, 170 2004, A\&A, 423, 335

Turner, D. G. 2003, JAVSO, 31, 160

Turner, D. G., Horsford, A. J., \& MacMillan, J. D. 1998, JRASC, 92, 320

Yang, X. H., Fu, J. N., \& Zha, Q. 2012, AJ, 144, 92

Zhou, A.-Y. 2002, ASPC, 259, 332 
Mohamed Abdel-Sabour and Ahmed Shokry: National Research Institute of Astronomy and Geophysics (NRIAG), 11421 Helwan, Cairo, Egypt (sabour2000@hotmail.com).

Ahmed Ibrahim: Dept. of Astronomy and Meteorology, Faculty of Science, Al-Azhar University, Nasr City, Cairo, Egypt.

Ahmed Ibrahim: King Saud Univ, Department of Physics \& Astronomy, Coll. Sci, Riyadh 11451, Saudi Arabia (ahabdalla@ksu.edu.sa). 